\title{
Multistep-Galerkin Methods for Hyperbolic Equations
}

\author{
By Vassilios A. Dougalis*
}

\begin{abstract}
Multistep methods for first- and second-order ordinary differential equations are used for the full discretizations of standard Galerkin approximations to the initial-periodic boundary value problem for first-order linear hyperbolic equations in one space dimension and to the initial-boundary value problem for second-order linear selfadjoint hyperbolic equations in many space dimensions. $L^{2}$-error bounds of optimal order in space and time are achieved for large classes of such multistep methods.
\end{abstract}

1. Introduction. In recent years there has been considerable interest in the numerical solution by Galerkin (finite element) methods of initial-boundary value problems for first-order systems of linear hyperbolic equations in one space variable and second-order linear hyperbolic equations in many space variables.

Usually, the continuous-time semidiscrete approximations and low-order (firstand second-order accurate) full discretizations in time have been considered. It is also possible to consider higher order accurate full discretizations in time in order to match the high order of accuracy achieved by the Galerkin method in the space discretization. Recently, Mock [15] has analyzed explicit fourth-order accurate Galerkin methods for the first-order case. For single-step Padé discretizations in time also in the first-order case cf. [18]. Crouzeix [3] and Baker and Bramble [2] have analyzed single-step fully discrete methods for the second-order hyperbolic case. Gekeler in [9] has analyzed the stability of multistep methods for second-order hyperbolic equations.

In this work as well as in [5] we analyze the stability and prove optimal $L^{2}$-error estimates both in space and time for multistep methods for first- and second-order hyperbolic equations.

In Section 2 we consider multistep discretizations of the simple first-order equation (2.1) with periodic boundary conditions. The "standard" variational formulation (2.1') in $\widetilde{H}^{1}$ is used for the Galerkin method in the space direction. (For "nonstandard" formulations see, e.g. [1] , [4], [5], [14], [15], [21].) It is well known, cf. [7] , [12], that the order of accuracy in the $L^{2}$-error bound for the continuous-time approximation is nonoptimal in the case of an arbitrary finite-dimensional subspace of $\widetilde{H}^{1}$. Specifically, Dupont has proved in [7] that the Hermite cubics on a uniform mesh

\footnotetext{
Received December 6, 1976; revised April 10, 1978 and May 9, 1978.

AMS (MOS) subject classifications (1970). Primary 65M15, 65N15; Secondary 65N30, $65 \mathrm{LO} 05$.

*Work partly supported by the Office of Naval Research while the author was a graduate student at Harvard University 
give a (nonoptimal) $O\left(h^{3}\right)$ accuracy that cannot be improved. However, Dupont, op. cit., has proved that the optimal accuracy is achieved for the piecewise linear continuous functions and cubic splines. The same result has been proved by Thomé [19] for the smooth splines of arbitrary order for the nonperiodic pure initial value problem. For the variable coefficient case cf. [20] and also [22], [8]. In [19] and [20] superconvergence at the nodes is also proved with suitable choices of initial conditions and/or suitable (quasi)interpolation. In the periodic case of constant coefficients that we are considering, the formulations of [19] or [20] lead to equivalent superconvergence results. We chose the Thomé-Wendroff approach of [20] for the space part of the approximation, and we show in Theorem 2.1 that the optimal exponent in the $L^{2}$-error bound is preserved (as well as the superconvergence optimal estimates), under typical "Courant number" restrictions on $\tau / h$, for a large class of multistep full discretizations, cf. [10, Chapter 5], of the Galerkin semidiscretization. A certain boundedness condition, introduced in Definition 2.1 as Condition I has to be satisfied by the coefficients $\gamma_{j}$, which are associated with the multistep method and defined by (2.9). We verify that the "optimal" (in the sense of Henrici [10, p. 233]) $k$-step methods for $k=1,2,4$ (respectively, of order of accuracy $2,4,6$ ) satisfy Condition I. For such methods we prove in Theorem 2.1 that if $S^{\mu}, \mu \geqslant 2$, is the space of the smooth periodic polynomial splines of order $\mu$ on a uniform mesh and if the order of the $k$-step method is $p \geqslant 1$, the estimate

$$
\max _{0 \leqslant n \leqslant J}\left\|u_{h}^{n}-u^{n}\right\|_{L^{2}(0,1)}=O\left(h^{\mu}+\tau^{p}\right)
$$

holds, where $u, u_{h}^{n}$ are the solutions of $\left(2.1^{\prime}\right),(2.11)$, respectively, provided the starting values $\left\{u_{h}^{j}\right\}_{j=0}^{k-1}$ are accurate enough, cf. Remark 2 after Theorem 2.1. We also prove the associated superconvergence estimates (2.16.1)-(2.16.2).

In [5] the optimal $L^{2}$ global estimate (1.1) was derived for all polynomial splines of odd degree, i.e., with $\mu=2 m, m \geqslant 1$, by an extension of the methods and the results that Dupont obtained for $m=1,2$ in [7].

In Section 3 we consider the initial-boundary value problem (with homogeneous Dirichlet boundary conditions) for the second-order linear hyperbolic equation with symmetric space-varying coefficients (3.1). For simplicity, we consider the homogeneous case. For the space part we use any finite-dimensional subspace $S_{h}$ of $\stackrel{\circ}{H}^{1}(\Omega)$ for which the usual approximation property (3.4) holds. For the time part we consider a well-known class of multistep methods for special second-order differential equations, described, e.g. in [10, Chapter 6], on which we impose a growth condition analogous to the one in the first-order case, Condition II in Definition 3.1. As in the first-order case, the "optimal" methods, cf. [10, p. 309 et seq.], for $k=2,4$ (respective order of accuracy 4,6 ) satisfy this condition. Under a stability restriction on the time step (cf. Lemma 3.1), which in the presence of inverse assumptions on $S_{h}$ becomes a restriction on the "Courant number" $\tau / h$, we prove in Theorem 3.1 that the estimate

$$
\max _{0 \leqslant n \leqslant J}\left\|u^{n}-u_{h}^{n}\right\|_{L^{2}(\Omega)}=O\left(h^{r}+\tau^{p}\right)
$$


holds, where $u, u_{h}^{n}$ are the solutions of (3.3) and (3.7), respectively, $p$ is the order of the multistep method, and $O\left(h^{r}\right)$ is the optimal accuracy achievable in $S_{h}$. We assume that the starting values $\left\{u_{h}^{j}\right\}_{j=0}^{k-1}$ are close to $H^{1}$-projections of the initial data; cf. [6]. See Section 3.2 for details on starting values.

In the work of Gekeler [9], where the stability of multistep methods for firstorder parabolic and second-order hyperbolic equations is analyzed, alternative conditions of stability are given. We remark that the methods which satisfy the hypotheses of [9, Theorem 3, pp. 543-544] will also satisfy as a consequence Condition II. This can be seen by finding estimates on the coefficients $\gamma_{j}$ in an analogous way to Henrici's proof of [10, Lemma 6.2, p. 312].

In what follows we introduce some notation about the function spaces that we shall use. Let $\Omega$ be a bounded open subset of $\mathbf{R}^{N}$ with boundary $\partial \Omega$, a generic point of which we denote by $x=\left(x_{1}, \ldots, x_{N}\right)$. For a nonnegative integer $m$ let $C^{m}(\Omega)$ be the space of real-valued functions whose derivatives up to order $m$ are continuous in $\Omega$. The Sobolev space $H^{m}(\Omega)$ is defined as the set

$$
H^{m}(\Omega)=\left\{v \in L^{2}(\Omega): D^{\alpha} v \in L^{2}(\Omega), \forall \alpha:|\alpha| \leqslant m\right\}
$$

where $\alpha=\left(\alpha_{1}, \ldots, \alpha_{N}\right), \alpha_{i}$ nonnegative integers, $D^{\alpha} v=\partial^{|\alpha|} v / \partial x_{1}^{\alpha} \partial_{2} x_{2}^{\alpha_{2}} \cdots \partial x_{N}^{\alpha_{N}}$ in the distributional sense and $|\alpha|=\alpha_{1}+\cdots+\alpha_{N}$. Denoting by $\|\cdot\|$ and $(\cdot, \cdot)$ the norm and inner product on $L^{2}(\Omega)$ we see that $H^{m}(\Omega)$ is a Hilbert space under the norm

$$
\|v\|_{m}=\left(\sum_{|\alpha| \leqslant m}\left\|D^{\alpha} v\right\|^{2}\right)^{1 / 2} .
$$

We let $\stackrel{\circ}{H}^{1}(\Omega)=\left\{v \in H^{1}(\Omega):\left.v\right|_{\partial \Omega}=0\right\}$.

If $\left(X,\|\cdot\|_{X}\right)$ is a Banach space and $p=2$ or $p=\infty, L^{p}(0, T ; X)$ (where $T>0$ ) will denote the Banach space of (classes of) strongly measurable functions $f:(0, T)$ $\rightarrow X$ such that

or

$$
\|f\|_{L^{2}(0, T ; X)}=\left(\int_{0}^{T}\|f(t)\|_{X}^{2} d t\right)^{1 / 2}<\infty
$$

$$
\|f\|_{L^{\infty}(0, T ; X)}=\underset{0<t<T}{\operatorname{ess} \sup }\|f(t)\|_{X}<\infty,
$$

respectively. When there is no possible ambiguity, $L^{p}(0, T ; X)$ will be denoted by $L^{p}(X)$. For properties of these spaces, see e.g. [13].

In Section 2 we shall restrict ourselves to the one-dimensional case $\Omega=(0,1)$. We shall denote by $\widetilde{L}^{2}$ the set of functions in $L_{\text {loc }}^{2}\left(\mathbf{R}^{1}\right)$ which are periodic of period 1 , and define $\|u\|=\|u\|_{L^{2}(0,1)}$ for $u \in \widetilde{L}^{2}$. We also denote by $\widetilde{H}^{m}$ the real Sobolev space of functions, which have $m$ derivatives (in the distributional sense) in $L_{\text {loc }}^{2}\left(\mathbf{R}^{1}\right)$ and are periodic of period 1. We let $\|u\|_{m}=\|u\|_{H^{m}(0,1)}$ for $u \in \widetilde{H}^{m}$.

\section{First-Order Hyperbolic Equations.}

2.1. The Problem and the Multistep-Galerkin Methods. We consider for simplicity the real, homogeneous, first-order hyperbolic problem with periodic initial and 
boundary conditions:

$$
\begin{cases}u_{t}+u_{x}=0 & \text { in }(0,1) \times(0, T] \\ u(x, 0)=u_{0}(x) & \text { in }[0,1]\end{cases}
$$

where $T>0$ and $u_{0}(\cdot), u(\cdot, t)$ are periodic functions of period 1 with $u_{0}$ given.

Obviously, (2.1) has the following variational formulation: we seek $u \in L^{2}\left(\widetilde{H}^{1}\right), u_{t} \in$ $L^{2}\left(\widetilde{L}^{2}\right)$ such that

$$
\left\{\begin{array}{l}
\left(u_{t}, v\right)+\left(u_{x}, v\right)=0 \quad \forall v \in \widetilde{H}^{1}, 0<t \leqslant T, \\
u(0) \text { given in } \widetilde{H}^{1} .
\end{array}\right.
$$

For $h>0$ let $S_{h}$ be a finite-dimensional subspace of $\widetilde{H}^{1}$ with basis $\left\{\Phi_{i}\right\}_{i=1}^{N}$. We seek $u_{h}$, the Galerkin approximation to the solution $u$ of $\left(2.1^{\prime}\right)$ as a differentiable map $u_{h}$ : $[0, T] \rightarrow S_{h}$ of the form

$$
u_{h}(x, t)=\sum_{i=1}^{N} c_{i}(t) \Phi_{i}(x), \quad(x, t) \in[0,1] \times[0, T],
$$

such that

$$
\left\{\begin{array}{l}
\left(\frac{\partial u_{h}}{\partial t}, v\right)+\left(\frac{\partial u_{h}}{\partial x}, v\right)=0 \quad \forall v \in S_{h}, 0<t \leqslant T \\
u_{h}(0) \text { given in } S_{h} .
\end{array}\right.
$$

Then (2.2) is equivalent to the system of O.D. E.'s

$$
\left\{\begin{array}{l}
G C^{\prime}(t)+S C(t)=0, \quad 0<t \leqslant T, \\
C(0) \text { given in } \mathbf{R}^{N}
\end{array}\right.
$$

where $C(t)=\left[c_{1}(t), \ldots, c_{N}(t)\right]^{T}$ and $G, S$ are the $N \times N$ matrices with elements

$$
G_{i j}=h^{-1}\left(\Phi_{i}, \Phi_{j}\right), \quad S_{i j}=h^{-1}\left(\Phi_{i}, \Phi_{j}^{\prime}\right) \text {. }
$$

Since $G$ is positive definite, $\left(2.2^{\prime}\right)$ possesses a unique solution $C(t), 0 \leqslant t \leqslant T$.

Henceforth, the finite-dimensional space $S_{h}$ that we will consider will be $S_{h}=$ $S^{\mu}$, the space of 1-periodic splines on $[0,1]$ of order $\mu \geqslant 2$ on a uniform mesh with mesh-length $h=1 / N, N$ integer. For properties of the smooth splines, see e.g. [16], [17], [20]. Let $\phi=\chi^{* \mu}$, where $\chi$ is the characteristic function of $[-1 / 2,1 / 2]$ and $\phi_{j}(x)=\phi\left(h^{-1} x-j\right)$. Then a basis for $S^{\mu}$ is given, cf. [20], by the restrictions to $[0,1]$ of the functions

$$
\Phi_{j}(x)=\sum_{l \in Z} \phi_{j+l N}(x), \quad 1 \leqslant j \leqslant N .
$$

A modified basis can be constructed by letting

$$
\widetilde{\phi}(x)=\sum_{j=-q}^{q} d_{j} \phi(x-j)
$$


for $N \geqslant \mu+2 q$ and then by defining the new basis to consist of the restrictions to $[0,1]$ of the functions

$$
\widetilde{\Phi}_{j}(x)=\sum_{l \in Z} \widetilde{\phi}_{j+l N}(x) .
$$

Let now $((\cdot, \cdot)),\|||\| \mid$ denote the $L^{2}$-inner product and norm on $(-\infty, \infty)$

$$
((u, v))=\int_{-\infty}^{\infty} u(x) v(x) d x, \quad\|u\|=((u, u))^{1 / 2} .
$$

Following [20], we define the following trigonometric polynomials

$$
\begin{aligned}
& g(\theta)=h^{-1} \sum_{l \in Z}\left(\left(\phi_{0}, \phi_{l}\right)\right) e^{-i l \theta}, \\
& \tilde{g}(\theta)=h^{-1} \sum_{l \in Z}\left(\left(\tilde{\phi}_{0}, \widetilde{\phi}_{l}\right)\right) e^{-i l \theta}, \\
& d(\theta)=\sum_{j=-q}^{q} d_{j} e^{-i j \theta} .
\end{aligned}
$$

In [20, pp. 1061-1062] the following is proved:

LEMMA 2.1. There exist positive constants $\underline{g}, \bar{g}$ such that for all real $\theta$

$$
0<\underline{g} \leqslant g(\theta) \leqslant \bar{g} .
$$

If $d(\theta) \neq 0$ for all real $\theta$, then there also exist positive constants $\underline{\tilde{g}}, \overline{\widetilde{g}}$ such that for all real $\theta$

$$
0<\underline{\tilde{g}} \leqslant \widetilde{g}(\theta) \leqslant \overline{\widetilde{g}}
$$

We denote now by $\langle\cdot, \cdot\rangle$ and $|\cdot|$, respectively, the Euclidean inner product and norm on $\mathbf{R}^{N}$. For $U, V \in \mathbf{R}^{N}$ we also define

$$
\langle U, V\rangle_{h}=h\langle U, V\rangle=h \sum_{j=1}^{N} U_{j} V_{j}, \quad|U|_{h}=\langle U, U\rangle_{h}^{1 / 2}=\sqrt{h}|U| .
$$

In addition to (2.3) we also define the $N \times N$ matrices $\widetilde{G}, \widetilde{S}$ corresponding to the modified basis functions $\widetilde{\Phi}_{j}$ as

$$
\widetilde{G}_{i j}=h^{-1}\left(\widetilde{\Phi}_{i}, \widetilde{\Phi}_{j}\right), \quad \widetilde{S}_{i j}=h^{-1}\left(\widetilde{\Phi}_{i}, \widetilde{\Phi}_{j}^{\prime}\right) .
$$

With the notation introduced in Lemma 2.1 the proof of the following can be found in [20, pp. 1061-1062].

LEMMA 2.2. For $N \geqslant \mu$ the matrix $G$ is a symmetric, positive definite cyclic matrix with eigenvalues $g(2 \pi l / N), 1 \leqslant l \leqslant N$, and, therefore, for every $V \in \mathbf{R}^{N}$

$$
\underline{g}|V|_{h}^{2} \leqslant\langle G V, V\rangle_{h} \leqslant \bar{g}|V|_{h}^{2} .
$$

If $N \geqslant \mu+2 q$ and $d(\theta) \neq 0$ for real $\theta$, the matrix $\widetilde{G}$ is a symmetric, positive definite cyclic matrix with eigenvalues $\tilde{g}(2 \pi l / N), 1 \leqslant l \leqslant N$, and therefore, for every $V \in \mathbf{R}^{N}$

$$
\underline{\tilde{g}}|V|_{h}^{2} \leqslant\langle\widetilde{G} V, V\rangle_{h} \leqslant \overline{\widetilde{g}}|V|_{h}^{2}
$$


Now let $D$ be the cyclic matrix defined for $N>2 q$ by its first row:

$$
\left[d_{0}, d_{-1}, \ldots, d_{-q}, 0, \ldots, 0, d_{q}, \ldots, d_{1}\right] .
$$

Then it is obvious that for $v \in S^{\mu}, v=\Sigma_{j=1}^{N} V_{j} \Phi_{j}=\Sigma_{j=1}^{N} \widetilde{V}_{j} \widetilde{\Phi}_{j}$, where $V=0 \widetilde{V}$. It is also easy to see that $\widetilde{G}=D^{*} G D, \widetilde{S}=D^{*} S D$.

The following is proved in $[20$, p. 1062]:

LEMMA 2.3. If $N>4 q$ and there exist constants $\underline{d}, \bar{d}$ such that

$$
0<\underline{d} \leqslant|d(\theta)| \leqslant \bar{d} \text { for all real } \theta,
$$

then

$$
\underline{d}|V|_{h} \leqslant|D V|_{h} \leqslant \bar{d}|V|_{h} \text { for all } V \in \mathbf{R}^{N} .
$$

Now, let $u$ be a 1 -periodic continuous function. Its $\widetilde{\phi}$-quasi-interpolant is defined as $Q_{\tilde{\phi}} u \in S^{\mu}$ by

$$
\left(Q_{\tilde{\phi}} u\right)(x)=\sum_{j=1}^{N} u(j h) \widetilde{\Phi}_{j}(x) .
$$

Thomée and Wendroff have proved in [20] that a particular choice of $d(\theta)$ (cf. Lemma 2.5 , p. 1063 of [20]) and of the starting condition in (2.2) as $u_{h}(0)=Q_{\tilde{\phi}} u_{0}$ leads in our case of (2.1), for a sufficiently smooth solution $u$, to the following error estimates for $0 \leqslant t \leqslant T$ :

$$
|\widetilde{C}(t)-U(t)|_{h}=O\left(h^{2 \mu}\right), \quad\left\|u_{h}(t)-Q_{\tilde{\phi}} u(t)\right\|=O\left(h^{2 \mu}\right), \quad\left\|u(t)-u_{h}(t)\right\|=O\left(h^{\mu}\right),
$$

where $u_{h}(t)$ is the solution of $(2.2)$,

$$
\begin{gathered}
u_{h}(t)=\sum_{j=1}^{N} \tilde{c}_{j}(t) \widetilde{\Phi}_{j}, \quad U(t)=[u(0, t), \ldots, u(j h, t), \ldots, u(1, t)]^{T}, \\
\widetilde{C}=\left[\tilde{c}_{1}, \ldots, \tilde{c}_{N}\right]^{T} .
\end{gathered}
$$

We establish now some notation and some basic concepts involved in a multistep method for first-order ordinary differential equations. Consider the problem

$$
\left\{\begin{array}{l}
y^{\prime}=f(y, t), \quad 0<t \leqslant T, \\
y(0)=y_{0},
\end{array}\right.
$$

where we assume that $f$ is a continuous function in both variables in the domain of its definition and Lipschitz continuous in $y$ uniformly in $t \in[0, T]$. Let $\tau>0$ be the discrete time step, and let $y^{n}$ denote the approximation to $y\left(t^{n}\right)$, where $t^{n}=n \tau$, $n=0,1,2, \ldots$ Let $J=T / \tau$, an integer for simplicity, and let $f^{n}=f\left(y^{n}, t^{n}\right)$. Then a general linear $k$-step method for the numerical solution of the first-order equation (2.5) is a scheme of the form

$$
\left\{\begin{array}{l}
\sum_{j=0}^{k} \alpha_{j} y^{n+j}=\tau \sum_{j=0}^{k} \beta_{j} f^{n+j}, \quad n=0,1,2, \ldots, J-k \\
\left\{y^{j}\right\}_{j=0}^{k-1} \quad \text { given starting values, }
\end{array}\right.
$$


where $k \geqslant 1$ is a fixed integer and where $\alpha_{j}, \beta_{j}, 0 \leqslant j \leqslant k$, are real constants independent of $n$. We shall assume hereafter that $\alpha_{k}>0$ and that $\left|\alpha_{0}\right|+\left|\beta_{0}\right|>0$. We associate with the difference equation (2.6) the polynomials of one complex variable $z$

$$
\left\{\begin{array}{l}
\rho(z)=\alpha_{k} z^{k}+\alpha_{k-1} z^{k-1}+\cdots+\alpha_{0}, \\
\sigma(z)=\beta_{k} z^{k}+\beta_{k-1} z^{k-1}+\cdots+\beta_{0} .
\end{array}\right.
$$

For definitions of stability, convergence and order of the multistep methods (2.6) cf. [10, Section 5.2]. It is well known that the order $p$ of a stable $k$-step method may be at most $k+2$ when $k$ is even and at most $k+1$ when $k$ is odd. A stable $k$-step method ( $k$-even) whose order is $k+2$ will be called an optimal $k$-step method. For the construction of such optimal methods see $[10$, p. 233 et seq.]

In association with (2.6) we introduce the complex-valued functions $\delta_{j}(\zeta)$ of a complex variable $\zeta$ given by the formula

$$
\delta_{j}(\zeta)=\frac{\alpha_{j}+\zeta \beta_{j}}{\alpha_{k}+\zeta \beta_{k}}, \quad 0 \leqslant j \leqslant k .
$$

An easy calculation gives

Lemma 2.4. Let $t \in[-K, K]$, where $K$ is a given positive number. Then $\delta_{j}($ it $)$ is bounded. If $\beta_{k} \neq 0$, then $\delta_{j}$ (it) is bounded for all real t. If $\beta_{k} \geqslant 0$ and $t \in[0, K]$, $\delta_{j}(t)$ is bounded. If $\beta_{k}>0$, then $\delta_{j}(t)$ is bounded for all $t>0$.

For $\zeta$ complex we consider the one-parameter family of polynomials of a complex variable $z$

$$
p(z, \zeta)=\delta_{k}(\zeta)+\delta_{k-1}(\zeta) z+\cdots+\delta_{0}(\zeta) z^{k}
$$

We define the coefficients $\left\{\gamma_{j}(\zeta)\right\}_{j=0}^{\infty}$ by the formal expansion

$$
[p(z, \zeta)]^{-1}=\gamma_{0}(\zeta)+\gamma_{1}(\zeta) z+\gamma_{2}(\zeta) z^{2}+\cdots
$$

Definition 2.1. We shall say that the $k$-step method (2.6) satisfies Condition I if there exists a positive $K$ such that the coefficients $\left\{\gamma_{j}(\zeta)\right\}_{j=0}^{\infty}$ defined by (2.9) satisfy the estimate

$$
\left|\gamma_{j}(i t)\right| \leqslant \Gamma \text { for all } j \text { and for all } t \in[-K, K],
$$

where $\Gamma$ is a constant that depends on the method (2.6) and $K$.

Condition I can also be interpreted in terms of the behavior of the zeros of the polynomial $p(z, i t)$ for $|t| \leqslant K$. In [5, pp. 3-40-3-46] we verify that it is satisfied by many frequently used optimal multistep methods for first-order equations. We furnish below some examples of such methods and the associated bounds $K$ as in Definition 2.1.

(a) $k=1$. The optimal $(p=2)$ stable single-step method is the implicit trapezoidal rule $[11$, p. 42$]$ given by the parameters $\alpha_{1}=1, \alpha_{0}=-1, \beta_{1}=\beta_{0}=1 / 2$. It satisfies Condition I for all $t \in \mathbf{R}^{\mathbf{1}}$.

(b) $k=2$. (i) The optimal ( $p=4)$ implicit, stable, 2-step method [10, p. 234], $\left[11\right.$, p. 42] is Simpson's rule given by the parameters $\alpha_{2}=1, \alpha_{1}=0, \alpha_{0}=-1$, 
$\beta_{2}=\beta_{0}=1 / 3, \beta_{1}=4 / 3$. Condition $I$ is satisfied with any $K \in(0, \sqrt{3})$.

(ii) One frequently used nonoptimal 2-step method is the explicit midpoint rule or leapfrog method $(p=2)$ which is given by $\alpha_{2}=1, \alpha_{1}=0, \alpha_{0}=-1, \beta_{2}=$ $\beta_{0}=0, \beta_{1}=2$ and satisfies Condition I with $0<K<1$.

(c) $k=4$. It is known [11, pp. 38-39] that there exists a one-parameter family of optimal $(p=6)$, stable, implicit 4-step methods. They are given by the parameters

$$
\begin{aligned}
& \alpha_{4}=1, \quad \alpha_{3}=-2 \lambda, \quad \alpha_{2}=0, \quad \alpha_{1}=2 \lambda, \quad \alpha_{0}=-1, \\
& \beta_{0}=\beta_{4}=(14+\lambda) / 45, \quad \beta_{1}=\beta_{3}=(64-34 \lambda) / 45, \\
& \beta_{2}=(8-38 \lambda) / 15,
\end{aligned}
$$

where $\lambda \in(-1,1)$. In [5, p. 3-34 et seq.] we verify computationally that these methods satisfy Condition I with $0<K(\lambda)<R(\lambda)$, where $R(\lambda)$ is given for some values of $\lambda$ by the following table:

\begin{tabular}{rlcl}
\hline \multicolumn{1}{c}{$\lambda$} & \multicolumn{1}{c}{$R(\lambda)$} & $\lambda$ & $R(\lambda)$ \\
\hline-1.0 & 0.0 & 0.1 & 1.5068 \\
-0.9 & 0.0903 & 0.2 & 1.5558 \\
-0.8 & 0.2466 & 0.3 & 1.5942 \\
-0.7 & 0.4273 & 0.4 & 1.6251 \\
-0.6 & 0.6165 & 0.5 & 1.6506 \\
-0.5 & 0.8020 & 0.6 & 1.6719 \\
-0.4 & 0.9742 & 0.7 & 1.6901 \\
-0.3 & 1.1269 & 0.8 & 1.7059 \\
-0.2 & 1.2561 & 0.9 & 1.7198 \\
-0.1 & 1.3610 & 1.0 & 0.0 \\
0.0 & 1.4433 & & \\
\hline
\end{tabular}

We define now the associated $k$-step Galerkin method for the solution of the initial value problem $\left(2.1^{\prime}\right)$. We seek $\left\{u_{h}^{n}\right\}_{n=0}^{J} \in S^{\mu}$ such that

$$
\left\{\begin{array}{l}
\left(\sum_{j=0}^{k} \alpha_{j} u_{h}^{n+j}, v\right)+\tau\left(\sum_{j=0}^{k} \beta_{j} u_{h, x}^{n+j}, v\right)=0, \quad 0 \leqslant n \leqslant J-k, \forall v \in S^{\mu}, \\
\left\{u_{h}^{j}\right\}_{j=0}^{k-1} \text { given in } S^{\mu} .
\end{array}\right.
$$

Writing

$$
u_{h}^{n}=\sum_{j=1}^{N} c_{j}^{n} \Phi_{j}=\sum_{j=1}^{N} \widetilde{c}_{j}^{n} \widetilde{\Phi}_{j},
$$

we see that $(2.11)$ is equivalent to the problem 


$$
\left\{\begin{array}{l}
\sum_{j=0}^{k}\left(\alpha_{j} G+\tau \beta_{j} S\right) C^{n+j}=0, \quad 0 \leqslant n \leqslant J-k, \\
\left\{C^{j}\right\}_{j=0}^{k-1} \text { given, }
\end{array}\right.
$$

where $C^{n}=\left[c_{1}^{n}, c_{2}^{n}, \ldots, c_{N}^{n}\right]^{T}$, or to

$$
\left\{\begin{array}{l}
\sum_{j=0}^{k}\left(\alpha_{j} \widetilde{G}+\tau \beta_{j} \widetilde{S}\right) \widetilde{C}^{n+j}=0, \quad 0 \leqslant n \leqslant J-k, \\
\left\{\widetilde{C}^{j}\right\}_{j=0}^{k-1} \text { given, }
\end{array}\right.
$$

where $\widetilde{C}^{n}=\left[\tilde{c}_{1}^{n}, \widetilde{c}_{2}^{n}, \ldots, \widetilde{c}_{N}^{n}\right]^{T}$. Of course $C^{n}=D \widetilde{C}^{n}$.

In practice we use (2.12) to advance in time since $G$ and $S$ will have, in general, smaller bandwidths than $\widetilde{G}$ and $\widetilde{S}$. Now since $\Phi_{i}, \Phi_{j}$ are 1-periodic functions, $S$ is antisymmetric. Hence, since $\alpha_{k}>0$ and $G$ is symmetric and positive definite, we conclude that the matrix $\alpha_{k} G+\tau \beta_{k} S$ is invertible. Hence, the solutions $C^{n}$ of the linear systems (2.12) exist uniquely, Under the hypotheses of Lemma 2.3 so do the solutions $\widetilde{C}^{n}$ of $\left(2.12^{\prime}\right)$. We shall discuss starting values in Section 2.2.

2.2. Error Estimates. We first present a preliminary lemma. To establish notation, let $\hat{v}(\xi)$ denote the Fourier transform of a function $v(x) \in L^{2}(-\infty, \infty)$

$$
\hat{v}(\xi)=\int_{-\infty}^{\infty} v(x) e^{-i x \xi} d x .
$$

We suppose that $d(\theta)$ is chosen so that the Fourier transform of the associated modified $\widetilde{\phi}(x)$ has the property that, for some integer $s$

$$
\hat{\tilde{\phi}}(\xi)=1+O\left(\xi^{s}\right) \quad \text { as } \xi \rightarrow 0 .
$$

We suppose that $s \geqslant \mu$ so that we have the approximation property for the $\widetilde{\phi}$-quasiinterpolant $Q_{\tilde{\phi}}$

$$
\left\|v-Q_{\widetilde{\phi}} v\right\| \leqslant C h^{\mu}\left\|\frac{\partial^{\mu} v}{\partial x^{\mu}}\right\| .
$$

For details cf. [20], [17]. In [20, Lemma 2.5] a particular $d(\theta)$ is constructed for which $s=2 \mu$

The following lemma can be proved by a specialization in the constant coefficient case for (2.1) of the general proof in [20, Lemmas 4.1 and 4.2]. The exponent $s$ in the estimate below is a consequence of (2.13). The proof in [20], which is given for $s=2 \mu$, can be easily modified to yield (2.14).

LEMmA 2.5. Let $\widetilde{\phi}$ satisfy (2.13) for some integer $s, \mu \leqslant s \leqslant 2 \mu$, let $u$ be the solution of (2.1), and suppose that for $0 \leqslant j \leqslant s+1$ and for every $t \in[0, T]$, $\partial^{j} u / \partial x^{j}$ are continuous, 1-periodic functions. 
Then there exists a constant $C$ independent of $h$ and $u$ such that

$$
\max _{1 \leqslant j \leqslant N, 0 \leqslant t \leqslant T}\left|\sum_{l=1}^{N}\left(\widetilde{G}_{j l} \frac{\partial u}{\partial t}(l h, t)+\widetilde{S}_{j l} u(l h, t)\right)\right| \leqslant C h^{s}\left\|\frac{\partial^{s+1} u}{\partial x^{s+1}}\right\|_{L^{\infty}\left(L^{\infty}\right)}
$$

We proceed now with the main error estimate. The algebraic manipulations follow the ordinary differential equation case treated by Henrici [10, Lemma 5.6, p. 243], and some of the analytic machinery is analogous to that used in the parabolic case by Zlámal [24, p. 355 et seq.].

TheOREM 2.1. Suppose that the $k$-step method (2.6) is of order $p \geqslant 1$ and that it satisfies Condition I for some $K>0$. Let $u_{h}^{n}=\Sigma_{j=1}^{N} c_{j}^{n} \Phi_{j}=\Sigma_{j=1}^{N} \widetilde{c}_{j}^{n} \widetilde{\Phi}_{j}$ be the solution of the $k$-step Galerkin method (2.12)-(2.12'). Let $\tilde{\phi}$ satisfy (2.13) for some integer $s, \mu \leqslant s \leqslant 2 \mu$. Let $u$, the solution of (2.1), satisfy $u \in L^{2}\left(\widetilde{H}^{p+1}\right)$ and the hypotheses of Lemma 2.5. Then, if

$$
0<\tau \max _{1 \leqslant i \leqslant N}\left|\lambda_{i}\right| \leqslant K,
$$

where $\lambda_{i}$ are the eigenvalues of the matrix $G^{-1} S$, we have that

$$
\begin{aligned}
& \max _{0 \leqslant n \leqslant J}\left|\widetilde{C}^{n}-U^{n}\right|_{h} \\
& \leqslant C_{1}\left\{\sum_{j=0}^{k-1}\left|\widetilde{C}^{j}-U^{j}\right|_{h}+h^{s}\left\|\frac{\partial^{s+1} u}{\partial x^{s+1}}\right\|_{L^{\infty}\left(L^{\infty}\right)}+\tau^{p}\left\|\frac{\partial^{p+1} u}{\partial t^{p+1}}\right\|_{L^{2}\left(\tilde{L}^{2}\right)}\right\} \\
& \max _{0 \leqslant n \leqslant J}\left\|u_{h}^{n}-Q_{\tilde{\phi}} u^{n}\right\| \\
& \leqslant C_{2}\left\{\sum_{j=0}^{k-1}\left\|u_{h}^{j}-Q_{\tilde{\phi}} u^{j}\right\|+h^{s}\left\|\frac{\partial^{s+1} u}{\partial x^{s+1}}\right\|_{L^{\infty}\left(L^{\infty}\right)}+\tau^{p}\left\|\frac{\partial^{p+1} u}{\partial t^{p+1}}\right\|_{L^{2}\left(\tilde{L}^{2}\right)}\right\} \\
& \max _{0 \leqslant n \leqslant J}\left\|u_{h}^{n}-u^{n}\right\| \leqslant C_{3}\left\{\sum_{j=0}^{k-1}\left\|u_{h}^{j}-Q_{\tilde{\phi}^{\prime}} u^{j}\right\|+h^{\mu}\left\|\frac{\partial^{\mu} u}{\partial x^{\mu}}\right\| \|_{L^{\infty}\left(\tilde{L}^{2}\right)}\right. \\
& \left.+h^{s}\left\|\frac{\partial^{s+1} u}{\partial x^{s+1}}\right\|_{L^{\infty}\left(L^{\infty}\right)}+\tau^{p}\left\|\frac{\partial^{p+1} u}{\partial t^{p+1}}\right\|_{L^{2}\left(\tilde{L}^{2}\right)}\right\}
\end{aligned}
$$

where $u^{n}=u(n \tau), U^{n}$ is the $N$-vector with components $u^{n}(j h)$ and where $C_{i}, i=$ $1,2,3$, are constants which do not depend on $h, \tau, u$ or $u_{h}$.

Proof. Let $E^{n}=\widetilde{C}^{n}-U^{n}$. Using $\left(2.12^{\prime}\right)$, we obtain that $E^{n}$ satisfies

$$
\sum_{j=0}^{k}\left(\alpha_{j} \widetilde{G}+\tau \beta_{j} \widetilde{S}\right) E^{n+j}=-\widetilde{G} R^{n}-\tau F^{n} \quad \text { for } 0 \leqslant n \leqslant J-k,
$$

where $R^{n}$ is the $N$-vector with components $r^{n}(j h)$, where

$$
r^{n}=\sum_{j=0}^{k} \alpha_{j} u^{n+j}-\tau \sum_{j=0}^{k} \beta_{j} u_{t}^{n+j}
$$


and

$$
F^{n}=\sum_{j=0}^{k} \beta_{j}\left(\widetilde{G} U_{t}^{n+j}+\widetilde{S} U^{n+j}\right) .
$$

Multiplying both sides of (2.17) by $\widetilde{G}^{-1}$ and defining

$$
G^{n}=-R^{n}-\tau \widetilde{G}^{-1} F^{n},
$$

we obtain, with $M=\widetilde{G}^{-1} \widetilde{S}$,

$$
\sum_{j=0}^{k}\left(\alpha_{j} I+\tau \beta_{j} M\right) E^{n+j}=G^{n}, \quad 0 \leqslant n \leqslant J-k .
$$

Since $\widetilde{G}$ is cyclic, so is $\widetilde{G}^{-1}$ and, therefore, $M$ is a cyclic, antisymmetric matrix. Hence, $\alpha_{k} I+\tau \beta_{k} M$ is invertible. Recalling now the definition (2.7) of $\delta_{j}$, we can rewrite the above as

$$
\sum_{j=0}^{k} \delta_{j}(\tau M) E^{n+j}=L^{n}
$$

where

$$
L^{n}=\left(\alpha_{k} I+\tau \beta_{k} M\right)^{-1} G^{n}
$$

Using (2.8) and equating powers of $z$ in (2.9), we obtain the identities

$$
\left\{\begin{array}{l}
\delta_{k} \gamma_{0}=1, \\
\delta_{k} \gamma_{j}+\delta_{k-1} \gamma_{j-1}+\cdots+\delta_{k-j} \gamma_{0}=0, \quad 1 \leqslant j \leqslant k \\
\delta_{k} \gamma_{l}+\delta_{k-1} \gamma_{l-1}+\cdots+\delta_{0} \gamma_{l-k}=0, \quad l \geqslant k
\end{array}\right.
$$

As in Henrici [10, p. 243] and Zlámal [24, p. 355] by considering (2.21) with $n-k-l$ instead of $n$, premultiplying both of its sides by $\gamma_{l}(\tau M)$, summing the resulting equations from $l=0$ to $l=n-k \geqslant 0$, rearranging and finally using the above identities, we obtain the solution of the difference equation (2.21) in terms of the $L^{n}$ and the $\left\{E^{j}\right\}_{j=0}^{k-1}$. Noting that for a $N \times N$ matrix $A,|A|=|A|_{h}$, we obtain the following estimate on $\left|E^{n}\right|_{h}$, for $k \leqslant n \leqslant J$ :

$$
\begin{gathered}
\left|E^{n}\right|_{h} \leqslant\left(\left|\gamma_{n-k}(\tau M)\right|\left|\delta_{k-1}(\tau M)\right|+\cdots+\left|\gamma_{n-2 k+1}(\tau M)\right|\left|\delta_{0}(\tau M)\right|\right)\left|E^{k-1}\right|_{h} \\
+\cdots+\left|\gamma_{n-k}(\tau M)\right|\left|\delta_{0}(\tau M)\right|\left|E^{0}\right|_{h}+\sum_{l=0}^{n-k}\left|\gamma_{l}(\tau M)\right|\left|L^{n-k-l}\right|_{h} .
\end{gathered}
$$

Since $M$ is antisymmetric it is normal, and we have that

$$
\left|\delta_{j}(\tau M)\right|=\max _{1 \leqslant i \leqslant N}\left|\delta_{j}\left(\tau \lambda_{i}\right)\right|, \quad\left|\gamma_{l}(\tau M)\right|=\max _{1 \leqslant i \leqslant N}\left|\gamma_{l}\left(\tau \lambda_{i}\right)\right|,
$$

where $\lambda_{i}, 1 \leqslant i \leqslant N$, are the (pure imaginary) eigenvalues of $M$, which coincide with the eigenvalues of the generalized eigenvalue problem $S V=\lambda G V$. Hence, using the 
stability restriction (2.15), Condition I (Definition 2.1) and Lemma 2.4 we conclude that

$$
\left|\delta_{j}(\tau M)\right| \leqslant C \quad \text { all } j \geqslant 0, \quad\left|\gamma_{l}(\tau M)\right| \leqslant \Gamma \quad \text { all } l \geqslant 0,
$$

where $C$ and $\Gamma$ are nonnegative constants that depend on the multistep method (2.6). Then (2.23) gives

$$
\max _{0 \leqslant n \leqslant J}\left|E^{n}\right|_{h} \leqslant C\left\{\sum_{j=0}^{k-1}\left|E^{j}\right|_{h}+\sum_{n=0}^{J-k}\left|L^{n}\right|_{h}\right\} .
$$

Now, since $M$ is antisymmetric and $\alpha_{k}>0$ we have by (2.22) that

$$
\left|L^{n}\right|_{h} \leqslant\left|\left(\alpha_{k} I+\tau \beta_{k} M\right)^{-1}\right|\left|G^{n}\right|_{h} \leqslant \frac{1}{\alpha_{k}}\left|G^{n}\right|_{h} .
$$

Hence, by (2.20)

$$
\left|L^{n}\right|_{h} \leqslant \frac{1}{\alpha_{k}}\left(\left|R^{n}\right|_{h}+\tau\left|\widetilde{G}^{-1}\right|\left|F^{n}\right|_{h}\right) .
$$

Now, since $Q_{\tilde{\phi}^{r}} r^{n}=\Sigma_{j=1}^{N} r^{n}(j h) \widetilde{\Phi}_{j}$, we obtain, using Lemma 2.2,

$$
\left\|Q_{\tilde{\phi}} r^{n}\right\|=\left\|\sum_{j=1}^{N} r^{n}(j h) \widetilde{\Phi}_{j}\right\|=\left\langle\widetilde{G}^{n}, R^{n}\right\rangle_{h}^{1 / 2} \geqslant \underline{\tilde{g}}^{1 / 2}\left|R^{n}\right|_{h} .
$$

And for $h$ sufficiently small, Lemma 2.2 gives that for some constant $C,\left\|Q_{\tilde{\phi}} r^{n}\right\| \leqslant$ $C\left\|r^{n}\right\|$. Hence, we obtain that $\left|R^{n}\right|_{h} \leqslant C\left\|r^{n}\right\|$. Using (2.18), Taylor's theorem with remainder and the fact that our $k$-step method is of order $p$, we have that

$$
\begin{aligned}
r^{n}(x)= & \sum_{j=0}^{k} \alpha_{j} \frac{1}{p !} \int_{n \tau}^{(n+j) \tau}[(n+j) \tau-s]^{p} \frac{\partial^{p+1} u}{\partial t^{p+1}}(x, s) d s \\
& -\tau \sum_{j=0}^{k} \beta_{j} \frac{1}{(p-1) !} \int_{n \tau}^{(n+j) \tau}[(n+j) \tau-s]^{p-1} \frac{\partial^{p+1} u}{\partial t^{p+1}}(x, s) d s,
\end{aligned}
$$

from which, easily,

$$
\left\|r^{n}\right\|^{2} \leqslant C \tau^{2 p+1} \int_{n \tau}^{(n+k) \tau}\left\|\frac{\partial^{p+1} u(s)}{\partial t^{p+1}}\right\|^{2} d s .
$$

Hence, we have,

$$
\sum_{n=0}^{J-k}\left|R^{n}\right|_{h} \leqslant C \tau^{p}\left\|\frac{\partial^{p+1} u}{\partial t^{p+1}}\right\|_{L^{2}\left(\tilde{L}^{2}\right)} .
$$

Now, since $\left|F^{n}\right|_{h} \leqslant \max _{1 \leqslant j \leqslant N}\left|F_{j}^{n}\right|$, we obtain by (2.19) and Lemma 2.5 that

$$
\max _{0<n<J}\left|F^{n}\right|_{h} \leqslant C h^{s}\left\|\frac{\partial^{s+1} u}{\partial x^{s+1}}\right\|_{L^{\infty}\left(L^{\infty}\right)} .
$$

and, finally, since $\left|\widetilde{G}^{-1}\right| \leqslant \underline{\tilde{g}}^{-1}$ by Lemma 2.2 , we obtain that

$$
\tau\left|\widetilde{G}^{-1}\right| \sum_{n=0}^{J-k}\left|F^{n}\right|_{h} \leqslant C h^{s}\left\|\frac{\partial^{s+1} u}{\partial x^{s+1}}\right\|_{L_{\left(L^{\infty}\right)}^{\infty}} .
$$


Finally, using (2.24) - (2.27) we obtain (2.16.1). Now (2.16.2) follows from (2.16.1), the fact that for

$$
v=\sum_{j=1}^{N} \widetilde{V}_{j} \widetilde{\Phi}_{j} \in S^{\mu}, \quad\|v\|^{2}=\langle\widetilde{G} \widetilde{V}, \widetilde{V}\rangle_{h},
$$

and Lemma 2.2. Finally, since

$$
\left\|u_{h}^{n}-u^{n}\right\| \leqslant\left\|u_{h}^{n}-Q_{\tilde{\phi}} u^{n}\right\|+\left\|u^{n}-Q_{\tilde{\phi}} u^{n}\right\|,
$$

using $\left(2.13^{\prime}\right)$, we obtain (2.16.3).

Remark 1. The stability condition (2.15) requires that $\tau=O(h)$, because the eigenvalues $\lambda_{j}=\lambda_{j}\left(G^{-1} S\right)$ satisfy $\left|\lambda_{j}\right|=O\left(h^{-1}\right)$. To see that, let $i \lambda$, with $\lambda$ real, be an eigenvalue of the generalized eigenvalue problem $S V=i \lambda G V$, where $V=$ $\left[V_{1}, \ldots, V_{N}\right]^{T} \in \mathrm{C}^{N}$ is an associated eigenvector. Let $\bar{z}$ denote the complex conjugate of $z$. Then the above becomes

$$
\sum_{l=1}^{N} S_{j l} \bar{V}_{l}=-i \lambda \sum_{l=1}^{N} G_{j l} \bar{V}_{l} .
$$

Multiplying the above by $V_{j}$ for $1 \leqslant j \leqslant N$ and summing, we obtain that

$$
\sum_{j, l=1}^{N} V_{j} \bar{V}_{l}\left(\Phi_{j}, \Phi_{l}^{\prime}\right)=-i \lambda \sum_{j, l=1}^{N} V_{j} \bar{V}_{l}\left(\Phi_{j}, \Phi_{l}\right),
$$

from which with $w=\Sigma_{j=1}^{N} V_{j} \Phi_{j}$,

$$
\left(w, \bar{w}^{\prime}\right)=-i \lambda(w, \bar{w}) .
$$

Now let $w=w_{1}+i w_{2}$, with $w_{i} \in S^{\mu}, i=1,2$. Then from the above it follows that

$$
\lambda=2\left(w_{1}^{\prime}, w_{2}\right) /\left(\left\|w_{1}\right\|^{2}+\left\|w_{2}\right\|^{2}\right) .
$$

Now, since $w_{1} \in S^{\mu}$, it satisfies the inverse property $\left\|w_{1}^{\prime}\right\| \leqslant C h^{-1}\left\|w_{1}\right\|$, for some constant $C$. Hence, $|\lambda| \leqslant C h^{-1}$. Because of the cyclic structure of $G, S$, it is easy to obtain explicit formulas for the eigenvalues $\lambda_{j}$ of $G^{-1} S$. We immediately obtain that

$$
\lambda_{j}=\lambda_{j}\left(G^{-1} S\right)=\lambda_{j}(S) / \lambda_{j}(G) \text {. }
$$

Let $\left[g_{1}, g_{2}, \ldots, g_{\mu}, 0, \ldots, 0, g_{\mu}, g_{\mu-1}, \ldots, g_{2}\right]$ denote the first row of $G$, for $N \geqslant 2 \mu-1$. Then it is well known from the theory of cyclic matrices that the eigenvalues of $G$ are given by the formula

$$
\lambda_{j}(G)=g_{1}+2 \sum_{l=2}^{\mu} g_{l} \cos (l-1) \theta_{j}, \quad \theta_{j}=2 \pi j / N .
$$

Similarly, if $\left[0, s_{2}, s_{3}, \ldots, s_{\mu}, 0, \ldots, 0,-s_{\mu},-s_{\mu-1}, \ldots,-s_{2}\right]$ denotes the first row of $S$, we obtain that

$$
\lambda_{j}(S)=2 i \sum_{l=2}^{\mu} s_{l} \sin (l-1) \theta_{j}, \quad \theta_{j}=2 \pi j / N .
$$

With the definition (2.3) $g_{l}=O(1)$ and $s_{l}=O(1 / h)$. 
These formulas can be used then to find the stability bounds on $\tau / h$ from (2.15).

Remark 2. The starting values $\left\{u_{h}^{j}\right\}_{j=0}^{k-1}$ can, in principle, be obtained by a singlestep method (e.g. a Padé method) selected so that the accuracy in the error estimates (2.16) is recovered. Alternatively, define

$$
u_{*}^{0}=u_{0}, \quad u_{*}^{j}=\sum_{l=0}^{p-1} \frac{(j \tau)^{l}}{l !} \frac{\partial^{l} u(0)}{\partial t^{l}}, \quad 1 \leqslant j \leqslant k-1,
$$

so that $u_{*}^{j}=u(j \tau)+O\left(\tau^{p}\right)$.

Noting that

$$
\frac{\partial^{l} u(0)}{\partial t^{l}}=(-1)^{l} \frac{\partial^{l} u_{0}}{\partial x^{l}}
$$

and defining $u_{h}^{j}=Q_{\tilde{\phi}} u_{*}^{j}, 0 \leqslant j \leqslant k-1$, we see that the accuracy in the superconvergence estimates (2.16.1) and (2.16.2) is conserved. The $O\left(h^{\mu}+\tau^{p}\right)$ global $L^{2}$ estimate of (2.16.3) can be achieved by defining $u_{h}^{j}$ as the $L^{2}$-projections on $S^{\mu}$ of $u_{*}^{j}$

$$
\left(u_{h}^{j}, v\right)=\left(u_{*}^{j}, v\right) \quad \forall v \in S^{\mu}
$$

and then noting that

$$
\left\|u_{h}^{j}-Q_{\tilde{\phi}^{\prime}} u^{j}\right\| \leqslant\left\|u_{h}^{j}-u_{*}^{j}\right\|+\left\|u_{*}^{j}-u^{j}\right\|+\left\|u^{j}-Q_{\tilde{\phi}} u^{j}\right\|=O\left(h^{\mu}+\tau^{p}\right) .
$$

\section{Second-Order Hyperbolic Equations.}

3.1. The Problem and the Multistep-Galerkin Methods. In this section we shall be interested in approximating the solution of the following initial-boundary value problem. (To simplify matters we consider the homogeneous case.) Given $T, 0<T$ $<\infty$, we seek a function $u(x, t)$ defined on $\bar{\Omega} \times[0, T]$ (where $\Omega$ is a bounded open subset of $\mathbf{R}^{N}$ with sufficiently smooth boundary $\partial \Omega$ ) and satisfying

$$
\left\{\begin{array}{l}
\frac{\partial^{2} u}{\partial t^{2}}-\sum_{i, j=1}^{N} \frac{\partial}{\partial x_{i}}\left(a_{i j}(x) \frac{\partial u}{\partial x_{j}}\right)=0 \text { in } \Omega \times(0, T] \\
u=0 \text { on } \partial \Omega \times[0, T] \\
u(x, 0)=u_{0}(x) \text { in } \Omega \\
\frac{\partial u}{\partial t}(x, 0)=u_{1}(x) \text { in } \Omega .
\end{array}\right.
$$

It is assumed that $a_{i j}(x) \in C^{0}(\bar{\Omega})$ and that $a_{i j}(x)=a_{j i}(x)$ for $x \in \bar{\Omega}$ and that there exist constants $\alpha, A>0$ such that

$$
\alpha \sum_{i=1}^{N} \xi_{i}^{2} \leqslant \sum_{i, j=1}^{N} a_{i j}(x) \xi_{i} \xi_{j} \leqslant A \sum_{i=1}^{N} \xi_{i}^{2} \quad \forall x \in \bar{\Omega}, \xi=\left(\xi_{1}, \ldots, \xi_{N}\right) \in \mathbf{R}^{N}
$$

Let $a(\cdot, \cdot)$ be the bilinear form associated with problem (3.1)

$$
a(u, v)=\sum_{i, j=1}^{N} \int_{\Omega} a_{i j}(x) \frac{\partial u}{\partial x_{i}} \frac{\partial v}{\partial x_{j}} d x \quad \text { for } u, v \in H^{1}(\Omega) .
$$


Then the initial-boundary value problem (3.1) has the following weak formulation: Given $a_{i j} \in C^{0}(\bar{\Omega})$ and $u_{0}, u_{1} \in \stackrel{\circ}{H}^{1}(\Omega)$, find $u \in L^{2}\left(0, T ; \stackrel{\circ}{H}^{1}(\Omega)\right)$ such that $\partial^{2} u / \partial t^{2}$ $\in L^{2}\left(0, T ; H^{-1}(\Omega)\right)$ and satisfying

$$
\left\{\begin{array}{l}
\left(\frac{\partial^{2} u}{\partial t^{2}}, v\right)+a(u, v)=0 \quad \forall v \in \stackrel{\circ}{H}^{1}(\Omega), 0<t \leqslant T, \\
u(0)=u_{0}, \quad u_{t}(0)=u_{1} .
\end{array}\right.
$$

Henceforth, we assume that (3.3) has a unique solution $u$. In appropriate places below precise conditions on the smoothness of $u$, which guarantee the convergence results, will be imposed.

Let $h$ be a real parameter, $0<h \leqslant 1$ and let $S_{h}$ be a finite-dimensional subspace of $\stackrel{\circ}{H}^{1}(\Omega)$ which satisfies the following approximation property for $h$ sufficiently small, and integer $r \geqslant 1$ :

$$
\inf _{\phi \in S_{h}}\|f-\phi\|_{j} \leqslant C h^{r-j}\|f\|_{r}, \quad j=0,1,
$$

for all $f \in H^{r}(\Omega) \cap \stackrel{\circ}{H}^{1}(\Omega)$ and where $C$ does not depend on $h$ or $f$.

Multistep methods for the numerical solution of initial value problems for a special class of second-order differential equations are well known. See e.g. Henrici [10, Chapter 6] or Lambert [11, Chapter 9]. We consider the problem

$$
\begin{cases}y^{\prime \prime}=f(y, t), & 0<t \leqslant T, \\ y(0)=y_{0}, & y^{\prime}(0)=y_{1},\end{cases}
$$

where we assume that $f$ is a continuous function in both variables in the domain of its definition and Lipschitz continuous in $y$ uniformly in $t \in[0, T]$. This is a special class of second-order ordinary differential equations in the sense that no first-order derivatives $y^{\prime}$ appear explicitly as arguments of $f$. With the same notation as in Section 2 we say that a special $k$-step method for the numerical solution of the second-order equation (3.5) is a scheme of the form

$$
\left\{\begin{array}{l}
\sum_{j=0}^{k} \alpha_{j} y^{n+j}=\tau^{2} \sum_{j=0}^{k} \beta_{j} f^{n+j}, \quad n=0,1, \ldots, J-k, J \tau=T, \\
\left\{y^{j}\right\}_{j=0}^{k-1} \text { given starting values, }
\end{array}\right.
$$

with $k \geqslant 1$ and $\alpha_{j}, \beta_{j}, 0 \leqslant j \leqslant k$, real constants independent of $n$. Again we assume for definiteness that $\alpha_{k}>0$ and $\left|\alpha_{0}\right|+\left|\beta_{0}\right|>0$. We also assume that $\beta_{k} \geqslant 0$. With the difference equation (3.6) we associate again the polynomials $\rho(z)$ and $\sigma(z)$ defined by $\left(2.6^{\prime}\right)$. For the convergence, stability and order $p$ of such methods see [10, Section 6.1]. It is well known that the order $p$ of a stable special $k$-step method cannot exceed $k+2$. Again, it may be at most $k+2$ when $k$ is even and at most $k+1$ when $k$ is odd. A stable special $k$-step method ( $k$ even) whose order is $k+2$ will be called a special optimal $k$-step method. For the construction of such optimal methods see [10, p. 309, 
et seq.]. In association with (3.6) we consider again the functions $\delta_{j}, \gamma_{l}$ and $p$ defined by (2.7), (2.9), (2.8), respectively.

Definition 3.1. We shall say that the special $k$-step method (3.6) satisfies Condition II if there exists a positive $K$ such that the coefficients $\left\{\gamma_{j}(t)\right\}_{j=0}^{\infty}$ satisfy the estimate

$$
\left|\gamma_{j}(t)\right| \leqslant \Gamma j+\gamma \text { for all } j \text { and all } t \in[0, K] \text {, }
$$

where $\Gamma$ and $\gamma$ are positive constants depending on the method (3.6) and $K$.

In [5, pp. 4-20-4-27] we verify that Condition II is satisfied by many frequently used special optimal multistep methods for second-order equations. Examples of such methods and their associated bounds $K$ are

(a) $k=2$. For $\theta \geqslant 0$ we consider the one-parameter family of stable special 2-step methods given by $\alpha_{0}=\alpha_{2}=1, \alpha_{1}=-2, \beta_{0}=\beta_{2}=\theta, \beta_{1}=1-2 \theta$. For $\theta \neq$ $1 / 12$ the methods are of order $p=2$. For $\theta=1 / 12$ we obtain the St $\phi$ rmer-Numerov formula $[11$, p. 255] which is of order $p=4$. This is the only optimal special 2-step method [10, p. 311], [11, p. 256]. For $\theta=0$ we obtain an explicit method and for $\theta=1 / 4$ we obtain a 2 -step method of accuracy $p=2$ analyzed in $[6$, p. 886 et seq. $]$. See also [9]. For these methods we find that

$$
\left|\gamma_{l}(t)\right| \leqslant l+1, \quad l=0,1,2, \ldots \text { for } \begin{cases}0 \leqslant t \leqslant 4 /(1-4 \theta) & \text { if } 0 \leqslant \theta<1 / 4, \\ 0 \leqslant t<\infty & \text { if } \theta \geqslant 1 / 4 .\end{cases}
$$

Hence, Conditon II is satisfied with $K$ defined as above.

(b) $k=3$. The optimal implicit 3-step methods $(p=4)$ form a one-parameter family of methods and are given in [11, p. 256]. It turns out that these methods are linear combinations of two subsequent cycles of application of the 2-step StormerNumerov formula and that the stability restriction on $K$ is not improved as compared with the restriction required for the Stbrmer-Numerov formula.

(c) $k=4$. There exists a two-parameter family of 4-step methods of $p=5$ or 6 given by the formulas $[11$, p. 256]

$$
\begin{aligned}
& \alpha_{4}=1, \quad \alpha_{3}=-2-a, \quad \alpha_{2}=1+2 a+b, \quad \alpha_{1}=-a-2 b, \quad \alpha_{0}=b, \\
& \beta_{4}=(19+a-b) / 240, \quad \beta_{3}=(51-6 a+b) / 60, \\
& \beta_{2}=(7-97 a+7 b) / 120, \quad \beta_{1}=(1-6 a+51 b) / 60, \quad \beta_{0}=(-1+a+19 b) / 240 .
\end{aligned}
$$

If $b \neq 1$, then $p=5$; if $b=1$ then $p=6$. The methods are stable for $-1<$ $b \leqslant 1,-1-b \leqslant a<1+b$. Thus, the optimal methods $(p=6)$ are given by $b=1$, $-2 \leqslant a<2$. The optimal methods satisfy Condition II with the exception of the case $b=1, a=-2$. The associated bounds are

$$
K(a)=60(a+2) /(9 a+22), \quad-2<a<2 .
$$

We define now an associated special $k$-step Galerkin method for the solution of the initial-boundary value problem (3.3). We seek $\left\{u_{h}^{n}\right\}_{n=0}^{J} \in S_{h}$ such that 


$$
\left\{\begin{array}{l}
\left(\sum_{j=0}^{k} \alpha_{j} u_{h}^{n+j}, \phi\right)+\tau^{2} a\left(\sum_{j=0}^{k} \beta_{j} u_{h}^{n+j}, \phi\right)=0, \quad 0 \leqslant n \leqslant J-k, \forall \phi \in S_{h}, \\
\left\{u_{h}^{j}\right\}_{j=0}^{k-1} \text { given in } S_{h} .
\end{array}\right.
$$

Writing as usual

$$
u_{h}^{n}(x)=\sum_{i=1}^{M} c_{i}^{n} \phi_{i}(x)
$$

where $\left\{\phi_{i}\right\}_{i=1}^{M}$ is a basis of $S_{h}$, we see that (3.7) is equivalent to

$$
\left\{\begin{array}{l}
\sum_{j=0}^{k}\left(\alpha_{j} G+\tau^{2} \beta_{j} A\right) C^{n+j}=0, \quad 0 \leqslant n \leqslant J-k \\
\left\{C^{j}\right\}_{j=0}^{k-1} \text { given, }
\end{array}\right.
$$

where $C^{n}=\left[c_{1}^{n}, \ldots, c_{M}^{n}\right]^{T}$, and $G, A$ are the $M \times M$ matrices given by

$$
G_{i j}=\left(\phi_{i}, \phi_{j}\right), \quad A_{i j}=a\left(\phi_{i}, \phi_{j}\right) .
$$

The solutions $C^{n}, 0 \leqslant n \leqslant J$, of the linear systems (3.8) exist and are unique since (3.2) and $\alpha_{k}>0, \beta_{k} \geqslant 0$ imply the positive definiteness of the matrix $\alpha_{k} G+\tau^{2} \beta_{k} A$. We shall discuss starting values in Section 3.2.

3.2. The Error Estimate. We establish first an a priori estimate for the solutions of a difference equation associated with (3.6). The algebraic and analytic tools follow [10, Section 6.2] and [24].

Lemma 3.1. Let $f^{n} \in L^{2}(\Omega)$ for $0 \leqslant n \leqslant J$, and let $\left\{v^{j}\right\}_{j=0}^{k-1}$ be given functions in $S_{h}$. Given a special k-step method of the form (3.6) which satisfies Condition II for some $K>0$, let $\left\{v^{j}\right\}_{j=0}^{J} \in S_{h}$ satisfy

(3.9) $\left(\sum_{j=0}^{k} \alpha_{j} v^{n+j}, \phi\right)+\tau^{2} a\left(\sum_{j=0}^{k} \beta_{j} v^{n+j}, \phi\right)=\left(f^{n}, \phi\right), \quad 0 \leqslant n \leqslant J-k, \forall \phi \in S_{h}$.

Then for

$$
0<\tau^{2} \max _{1 \leqslant i \leqslant M}\left|\lambda_{i}\right| \leqslant K,
$$

where $\lambda_{i}$ are the eigenvalues of the matrix $G^{-1} A$, we have that

$$
\max _{0 \leqslant n<J}\left\|v^{n}\right\| \leqslant \frac{C}{\tau}\left\{\sum_{j=0}^{k-1}\left\|v^{j}\right\|+\sum_{n=0}^{J-k}\left\|f^{n}\right\|\right\},
$$

where the constant $C$ does not depend on $v^{n}, f^{n}, \tau$ or $h$.

Proof. As in Zlámal $\left[24\right.$, p. 355], let $\Phi(x)=\left[\phi_{1}(x), \ldots, \phi_{M}(x)\right]^{T}$, where $\left\{\phi_{i}(x)\right\}_{i=1}^{M}$ is the basis of $S_{h}$, and $W=G^{-1 / 2} \Phi$. Then the coordinates $w_{i}$ of $W$ form a basis for $S_{h}$. With notation introduced in Section 2.1, since $v^{n} \in S_{h}$, let $v^{n}=$ $\left\langle V^{n}, W\right\rangle$. It is easy to see that $\left(w_{i}, w_{j}\right)=\delta_{i j}$ and that $a\left(w_{i}, w_{j}\right)=B_{i j}$, where $B$ is the symmetric, positive definite matrix defined by $B=G^{-1 / 2} A G^{-1 / 2}$. Let $F^{n}$ be the $M$-vector with coordinates $F_{i}^{n}=\left(f^{n}, w_{i}\right)$. Recalling the definition of $\delta_{j}(\zeta)$ by 
(2.7), we can rewrite (3.9) as

$$
\sum_{j=0}^{k} \delta_{j}\left(\tau^{2} B\right) V^{n+j}=G^{n}, \quad 0 \leqslant n \leqslant J-k,
$$

with $G^{n}=\left(\alpha_{k} I+\tau^{2} \beta_{k} B\right)^{-1} F^{n}$. Using the same techniques as in the proof of Theorem 2.1 , we estimate $V^{n}$ by

$$
\begin{aligned}
\left|V^{n}\right| \leqslant & \left(\left|\gamma_{n-k}\left(\tau^{2} B\right)\right|\left|\delta_{k-1}\left(\tau^{2} B\right)\right|+\cdots+\left|\gamma_{n-2 k+1}\left(\tau^{2} B\right)\right|\left|\delta_{0}\left(\tau^{2} B\right)\right|\right)\left|V^{k-1}\right| \\
& +\cdots+\left|\gamma_{n-k}\left(\tau^{2} B\right)\right|\left|\delta_{0}\left(\tau^{2} B\right)\right|\left|V^{0}\right| \\
& +\sum_{l=0}^{n-k}\left|\gamma_{l}\left(\tau^{2} B\right)\right|\left|G^{n-k-l}\right|, \quad k \leqslant n \leqslant J .
\end{aligned}
$$

Since $B$ is positive definite, (3.10), Condition II and Lemma 2.4 give

$$
\begin{aligned}
\left|\delta_{j}\left(\tau^{2} B\right)\right| & =\max _{1 \leqslant i \leqslant M}\left|\delta_{j}\left(\tau^{2} \lambda_{i}\right)\right| \leqslant C, \quad \text { all } j, \\
\left|\gamma_{l}\left(\tau^{2} B\right)\right| & =\max _{1 \leqslant i<M}\left|\gamma_{l}\left(\tau^{2} \lambda_{i}\right)\right| \leqslant \Gamma l+\gamma, \quad l \geqslant 0,
\end{aligned}
$$

for constants $C, \Gamma, \gamma$, where $\lambda_{i}$ are the eigenvalues of $G^{-1} A$ which coincide with the eigenvalues of $B$. Hence the above estimate becomes

$$
\begin{aligned}
\left|V^{n}\right| \leqslant & C\{\Gamma[(n-k)+\cdots+(n-2 k+1)]+k \gamma\}\left|V^{k-1}\right| \\
& +\cdots+(C \Gamma(n-k)+\gamma)\left|V^{0}\right|+\sum_{l=0}^{n-k}(\Gamma l+\gamma)\left|G^{n-k-l}\right| \text { for } k \leqslant n \leqslant J,
\end{aligned}
$$

which for $\tau$ sufficiently small, since $T=J \tau$, implies that there exists a constant $C$ independent of $\tau, h, V^{n}, G^{n}$ such that

$$
\left|V^{n}\right| \leqslant \frac{C}{\tau}\left\{\sum_{j=0}^{k-1}\left|V^{j}\right|+\sum_{l=0}^{n-k}\left|G^{n-k-l}\right|\right\} .
$$

Now, since $\left|\left(\alpha_{k} I+\tau^{2} \beta_{k} B\right)^{-1}\right| \leqslant \alpha_{k}^{-1}$, we see that $\left|G^{n}\right| \leqslant \alpha_{k}^{-1}\left|F^{n}\right| \leqslant \alpha_{k}^{-1}\left\|f^{n}\right\|$ as in $\left[24\right.$, p. 356]. Finally, since $v^{n} \in S_{h}$ we obtain that $\left\|v^{n}\right\|=\left|V^{n}\right|$ and (3.11) follows from (3.12).

Remark. The stability condition (3.10) will require a bound on $\tau / h$ (in case $K<\infty)$ if the elements of $S_{h}$ satisfy the inverse hypothesis

$$
\|\phi\|_{1} \leqslant C h^{-1}\|\phi\| \quad \forall \phi \in S_{h} .
$$

This is easily deduced from the fact that the $\lambda_{i}$ 's are the eigenvalues of the generalized eigenvalue problem

$$
A V=\lambda G V \text { for } V \in \mathbf{R}^{M} .
$$

Hence, if $v(x)=\Sigma_{i=1}^{M} V_{i} \phi_{i}(x) \in S_{h}$, we see that $\lambda=a(v, v) /\|v\|^{2}$; and the result follows from (3.13) and (3.10).

We now refer to a well-known (cf. e.g. [23]) result about the elliptic projection of the solution $u$ of (3.3). 
LEMma 3.2. Let $u$ be the solution of (3.3) and suppose that it satisfies $\partial^{l} u(t) / \partial t^{l} \in H^{r}(\Omega), 0 \leqslant t \leqslant T, l=0,1,2, \ldots$ For $0 \leqslant t \leqslant T$ we define $\omega_{h}(t) \in$ $S_{h} b y$

$$
a\left(\omega_{h}(t), \phi\right)=a(u(t), \phi) \quad \forall \phi \in S_{h} .
$$

Then for some constant $C$ independent of $h, u, \omega_{h}$ we have that

$$
\left\|\frac{\partial^{l}}{\partial t^{l}}\left(u-\omega_{h}\right)(t)\right\|_{j} \leqslant C h^{r-j}\left\|\frac{\partial^{l} u}{\partial t^{l}}\right\|_{r}
$$

for $j=0,1$ and $l=0,1,2, \ldots$

The main result of this section is

THEOREM 3.1. Suppose that the $k$-step method (3.6) is of order $p \geqslant 1$ and that it satisfies Condition II for some $K>0$. Let $\left\{u_{n}^{n}\right\}_{n=0}^{J}$ be the solution of the $k$-step Galerkin method (3.7). Let $u$, the solution of (3.3) satisfy the requirements

$$
u \in L^{\infty}\left(H^{r}\right), \quad \frac{\partial^{2} u}{\partial t^{2}} \in L^{2}\left(H^{r}\right), \frac{\partial^{p+2} u}{\partial t^{p+2}} \in L^{2}\left(L^{2}\right) .
$$

Then, under the stability condition (3.10) we have

$$
\begin{aligned}
\max _{0 \leqslant n<J}\left\|u_{h}^{n}-u^{n}\right\| & \\
\leqslant C\left\{\frac{1}{\tau} \sum_{j=0}^{k-1}\left\|u_{h}^{j}-\omega_{h}^{j}\right\|+h^{r}\left(\|u\|_{L^{\infty}\left(H^{r}\right)}\right.\right. & \left.+\left\|\frac{\partial^{2} u}{\partial t^{2}}\right\|_{L^{2}\left(H^{r}\right)}\right) \\
& \left.+\tau^{p}\left\|\frac{\partial^{p+2} u}{\partial t^{p+2}}\right\|_{L^{2}\left(L^{2}\right)}\right\},
\end{aligned}
$$

where $u^{n}=u(n \tau), \omega_{h}^{n}=\omega_{h}(n \tau)$ is defined in Lemma 3.2 and where $C$ is a constant which does not depend on $h, \tau, u$ or $u_{h}$.

Proof. We define $\omega_{h}(t)$ by (3.14) and set $\zeta^{n}=u_{h}^{n}-\omega_{h}^{n}, \eta(t)=u(t)-\omega_{h}(t)$.

Then by (3.3), (3.7) and (3.14) we have that $\forall \phi \in S_{h}$

$$
\begin{aligned}
\left(\sum_{j=0}^{k} \alpha_{j} \xi^{n+j}, \phi\right)+\tau^{2} a\left(\sum_{j=0}^{k} \beta_{j} \xi^{n+j}, \phi\right) \\
=-\left(\sum_{j=0}^{k} \alpha_{j} \omega_{h}^{n+j}, \phi\right)-\tau^{2} a\left(\sum_{j=0}^{k} \beta_{j} \omega_{h}^{n+j}, \phi\right) \\
=-\left(\sum_{j=0}^{k} \alpha_{j} \omega_{h}^{n+j}, \phi\right)-\tau^{2} a\left(\sum_{j=0}^{k} \beta_{j} u^{n+j}, \phi\right) \\
=\left(H^{n}, \phi\right)-\left(r^{n}, \phi\right), \quad 0 \leqslant n \leqslant J-k,
\end{aligned}
$$

where

$$
H^{n}=\sum_{j=0}^{k} \alpha_{j} \eta^{n+j}
$$


and

$$
r^{n}=\sum_{j=0}^{k} \alpha_{j} u^{n+j}-\tau^{2} \sum_{j=0}^{k} \beta_{j} u_{t t}^{n+j}
$$

Since $\zeta^{n} \in S_{h}$ we have, by Lemma 3.1 that

$$
\max _{0 \leqslant n \leqslant J}\left\|\zeta^{n}\right\|=C\left\{\frac{1}{\tau} \sum_{j=0}^{k-1}\left\|\zeta^{j}\right\|+\frac{1}{\tau} \sum_{n=0}^{J-k}\left\|H^{n}\right\|+\frac{1}{\tau} \sum_{j=0}^{J-k}\left\|r^{n}\right\|\right\} .
$$

By Taylor's theorem,

$$
\eta((n+j) \tau)=\eta(n \tau)+j \tau \frac{\partial \eta}{\partial t}(n \tau)+\int_{n \tau}^{(n+j) \tau}[(n+j) \tau-s] \frac{\partial^{2} \eta}{\partial t^{2}}(s) d s .
$$

By (3.17) and the consistency of our $k$-step method (which implies that $\Sigma_{j=0}^{k} \alpha_{j}=$ $\left.\Sigma_{j=0}^{k} j \alpha_{j}=0\right)$ we obtain that for $0 \leqslant n \leqslant J-k$,

$$
H^{n}=\sum_{j=0}^{k} \alpha_{j} \int_{n \tau}^{(n+j) \tau}[(n+j) \tau-s] \frac{\partial^{2} \eta}{\partial t^{2}}(s) d s
$$

from which, easily

$$
\left\|H^{n}\right\|^{2} \leqslant C \tau^{3} \int_{n \tau}^{(n+k) \tau}\left\|\frac{\partial^{2} \eta}{\partial t^{2}}\right\|^{2} d s
$$

where $C$ depends on the $\left\{\alpha_{j}\right\}_{j=0}^{k}$ and $k$. Therefore, by (3.15)

$$
\frac{1}{\tau} \sum_{n=0}^{J-k}\left\|H^{n}\right\| \leqslant \frac{C}{\tau^{3 / 2}}\left\{\sum_{n=0}^{J-k}\left\|H^{n}\right\|^{2}\right\}^{1 / 2} \leqslant C\left\|\frac{\partial^{2} \eta}{\partial t^{2}}\right\|_{L^{2}\left(L^{2}\right)} \leqslant C h^{r}\left\|\frac{\partial^{2} u}{\partial t^{2}}\right\|_{L^{2}\left(H^{r}\right)}
$$

We examine now the $r^{n}$ term. Using (3.18), Taylor's theorem with remainder and the fact that our special $k$-step method is of order $p$, we obtain

$$
\begin{aligned}
r^{n}= & \frac{1}{(p+1) !} \sum_{j=0}^{k} \alpha_{j} \int_{n \tau}^{(n+j) \tau}[(n+j) \tau-s]^{p+1} \frac{\partial^{p+2} u}{\partial t^{p+2}}(s) d s \\
& -\frac{\tau^{2}}{(p-1) !} \sum_{j=0}^{k} \beta_{j} \int_{n \tau}^{(n+j) \tau}[(n+j) \tau-s]^{p-1} \frac{\partial^{p+2} u}{\partial t^{p+2}}(s) d s
\end{aligned}
$$

from which

$$
\left\|r^{n}\right\|^{2} \leqslant C \tau^{2 p+3} \int_{n \tau}^{(n+k) \tau}\left\|\frac{\partial^{p+2} u}{\partial t^{p+2}}(s)\right\|^{2} d s,
$$

where $C$ depends on $p, k,\left\{\alpha_{j}\right\}_{j=0}^{k},\left\{\beta_{j}\right\}_{j=0}^{k}$. Hence

$$
\frac{1}{\tau} \sum_{n=0}^{J-k}\left\|r^{n}\right\| \leqslant \frac{C}{\tau^{3 / 2}}\left\{\sum_{n=0}^{J-k}\left\|r^{n}\right\|^{2}\right\}^{1 / 2} \leqslant C \tau^{p}\left\|\frac{\partial^{p+2} u}{\partial t^{p+2}}\right\|_{L^{2}\left(L^{2}\right)} .
$$

Finally, (3.19), (3.20), (3.21), and the fact that

$$
\begin{aligned}
\max _{0 \leqslant n<J}\left\|u^{n}-u_{h}^{n}\right\| & \leqslant \max _{0 \leqslant n \leqslant J}\left\|\eta^{n}\right\|+\max _{0 \leqslant n \leqslant J}\left\|\zeta^{n}\right\| \\
& \leqslant C h^{r}\|u\|_{L^{\infty}\left(H^{r}\right)}+\max _{0 \leqslant n \leqslant J}\left\|\zeta^{n}\right\|
\end{aligned}
$$

give the desired estimate (3.16). 
To preserve the $O\left(h^{r}+\tau^{p}\right)$ accuracy it is sufficient to choose the starting values $\left\{u_{h}^{j}\right\}_{j=0}^{k-1}$ such that

$$
\sum_{j=0}^{k-1}\left\|u_{h}^{j}-\omega_{h}^{j}\right\|=O\left(\tau h^{r}+\tau^{p+1}\right) .
$$

We can achieve this as follows: Let $u_{0} \in C^{p+2}(\Omega), u_{1} \in C^{p+1}(\Omega)$ and $a_{i j} \in C^{1}(\Omega)$. We define $u_{*}^{j}$ for $0 \leqslant j \leqslant k-1$ by

$$
u_{*}^{0}=u_{0}, \quad u_{*}^{j}=\sum_{l=0}^{p} \frac{(j \tau)^{l}}{l !} \frac{\partial^{l} u(0)}{\partial t^{l}}, \quad 1 \leqslant j \leqslant k-1,
$$

where we express $\partial^{l} u(0) / \partial t^{l}$ in terms of $u_{0}, u_{1}, a_{i j}$ and their derivatives using (3.1). We easily see that

$$
\left\|u(j \tau)-u_{*}^{j}\right\|_{s} \leqslant C \tau^{p+1}\left\|\frac{\partial^{p+1} u}{\partial t^{p+1}}\right\|_{L^{\infty}\left(H^{s}\right)}, \quad s \geqslant 0 .
$$

We set now $u_{h}^{j}=\omega_{*}^{j}$, where $\omega_{*}^{j} \in S_{h}$ is defined by

$$
a\left(\omega_{*}^{j}, \phi\right)=a\left(u_{*}^{j}, \phi\right) \quad \forall \phi \in S_{h} .
$$

This choice obviously satisfies (3.22).

Acknowledgement. The author would like to thank Professor G. A. Baker for suggesting the topic and Professors G. Birkhoff and G. A. Baker for many interesting discussions of the material.

Mathematics Department

The University of Tennessee

Knoxville, Tennessee 37916

1. G. A. BAKER, “A finite element method for first order hyperbolic equations," Math. Comp., v. 29, 1975 , pp. 995-1006.

2. G. A. BAKER \& J. H. BRAMBLE, Semidiscrete and Single Step Fully Discrete Approximations for Second Order Hyperbolic Equations, Rep. No. 22, Centre de Mathématiques Appliqueés de l'Ecole Polytechnique, Paris, 1977.

3. M. CROUZEIX, Sur l'Approximation des Équations Différentielles Opérationelles Linéaires par des Méthodes de Runge-Kutta, Thèse de Doctorat d'Etat, Université Paris VI, 1975.

4. J. E. DENDY, "Two methods of Galerkin type achieving optimum $L^{2}$ rates of convergence for first order hyperbolics," SIAM J. Numer. Anal., v. 11, 1974, pp. 637-653.

5. V. A. DOUGALIS, High Order Fully Discrete Galerkin Approximations to Hyperbolic Equations, Ph. D. Thesis, Harvard University, May 1976.

6. T. DUPONT, " $L^{2}$-estimates for Galerkin methods for second order hyperbolic equations," SIAM J. Numer. Anal, v. 10, 1973, pp. 880-889.

7. T. DUPONT, "Galerkin methods for first order hyperbolics: an example," SIAM J. Numer. Anal., v. 10, 1973, pp. 890-899.

8. G. FIX \& N. NASSIF, "On finite element approximations to time dependent problems," Numer. Math., v. 19, 1972, pp. 127-135.

9. E. GEKELER, "Linear multistep methods and Galerkin procedures for initial boundary value problems," SIAM J. Numer. Anal., v. 13, 1976, pp. 536-548.

10. P. HENRICI, Discrete Variable Methods in Ordinary Differential Equations, Wiley, New York, 1962.

11. J. D. LAMBERT, Computational Methods in Ordinary Differential Equations, Wiley, New York, 1973. 
12. P. LESAINT, "Finite element methods for symmetric hyperbolic equations," Numer. Math., v. 21, 1973, pp. 244-255.

13. J. L. LIONS \& E. MAGENES, Problèmes aux Limites Non Homogènes et Applications, Vols. I \& II, Dunod, Paris, 1968.

14. M. S. MOCK, "Projection methods with different trial and test spaces," Math. Comp., v. 30, 1976, pp. 400-416.

15. M. S. MOCK, "Explicit finite element schemes for first order symmetric hyperbolic systems," Numer. Math., v. 26, 1976, pp. 367-378.

16. I. J. SCHOENBERG, "Contributions to the problem of approximation of equidistant data by analytic functions," Parts A and B, Quart. Appl Math., v. 4, 1946, pp. 45-99, $112-141$.

17. G. STRANG, "The finite element method and approximation theory," in Numerical Solution of Partial Differential Equations II (Synspade 1970), ed. by B. Hubbard, Academic Press, New York, 1971 , pp. 547-583.

18. B. SWARTZ \& B. WENDROFF, "The relative efficiency of finite difference and finite element methods. I: Hyperbolic problems and splines," SIAM J. Numer. Anal., v. 11, 1974, pp. 979-993.

19. V. THOMÉE, "Convergence estimates for semi-discrete Galerkin methods for initial-value problems," in Numerische, insbesondere approximations-theoretische Behandlung von Funktionalgleichungen, ed. by R. Ansorge and W. Törnig, Lecture Notes in Math., vol. 333, Springer-Verlag, Berlin and New York, 1973, pp. 243-262.

20. V. THOMÉE \& B. WENDROFF, "Convergence estimates for Galerkin methods for variable coefficient initial value problems," SIAM J. Numer. Anal., v. 11, 1974, pp. 1059-1068.

21. L. WAHLBIN, "A modified Galerkin procedure with Hermite cubics for hyperbolic problems," Math. Comp., v. 29, 1975, pp. 978-984.

22. B. WENDROFF, On Finite Elements for Equations of Evolution, Rep. LA-DC-72-1220, Los Alamos, N. M., 1972.

23. M. F. WHEELER, "A priori $L_{2}$ error estimates for Galerkin approximations to parabolic partial differential equations," SIAM J. Numer. Anal., v. 10, 1973, pp. 723-759.

24. M. ZLÁMAL, "Finite element multistep discretizations of parabolic boundary value problems," Math. Comp., v. 29, 1975, pp. 350-359. 